\title{
A dual representation of gain-loss hedging for European claims in discrete time
}

\section{Mustafa Ç. Pınar}

To cite this article: Mustafa Ç. Pınar (2012) A dual representation of gain-loss hedging for European claims in discrete time, Optimization, 61:4, 361-372, DOI: 10.1080/02331934.2012.665053

To link to this article: $\underline{h t t p: / / d x . d o i . o r g / 10.1080 / 02331934.2012 .665053 ~}$

曲 Published online: 22 Mar 2012.

Submit your article to this journal $\pi$

山 Article views: 75

Q View related articles $₫$

4 Citing articles: 1 View citing articles 


\title{
A dual representation of gain-loss hedging for European claims in discrete time
}

\author{
Mustafa Ç. Pınar* \\ Department of Industrial Engineering, Bilkent University, 06800 Bilkent, Ankara, Turkey
}

(Received 2 December 2010; final version received 26 January 2012)

\begin{abstract}
Superhedging of European claims in incomplete markets is a well-studied problem. The superhedging value of a European claim is known to yield a price too large to be interesting in some cases. In this note, an alternative hedging strategy based on an expected gain-loss criterion is studied for European claims in infinite state space, discrete time financial markets. A dual representation for the gain-loss hedging value is obtained.
\end{abstract}

Keywords: European claims; pricing; gain-loss hedging; superhedging; martingales

\section{Introduction}

Superhedging of European claims consists in finding the smallest initial capital required to dominate a stochastic liability at a known future date as well as computing the portfolio weights at any given time to ensure such domination. The superhedging strategy operates by setting up a portfolio of traded instruments, and modifying the composition of this portfolio at discrete-time points so as to dominate the stochastic liability at the pre-determined future date. The present value of the superhedging portfolio constitutes the superhedging value of the stochastic future liability. The problem has been studied in depth by several authors (e.g. [9,18]). Our desktop reference for this article is the book by Föllmer and Schied [9].

It is well-known that the superhedging value can be unacceptably large for practical purposes [9]. To alleviate this problem and obtain a tighter price bound, we advocate a different criterion for hedging European claims in discrete time financial markets, based on the gain-loss criterion of Bernardo and Ledoit [2], which does not fit into the well-studied framework of coherent risk measures (see Chapter 4 of [9]) (we elaborate on this point later). The gain-loss criterion measures the expected value of positive terminal values (gain) of a portfolio as well as that of the negative (loss) terminal values. A portfolio process with a large gain-loss ratio could be acceptable to many investors in the absence of arbitrage. Based on this observation, we subscribe to the view that a 'fair' financial market should not only disallow arbitrage, but also should not offer opportunities with a large expected gain-to-loss ratio.

*Email: mustafap@bilkent.edu.tr

ISSN 0233-1934 print/ISSN 1029-4945 online 
Therefore, financial instruments can be priced so as not to allow large expected gainto-loss ratios. More precisely, price bounds are calculated so as not to allow portfolios with gain-loss ratios in excess of a preselected threshold $\lambda \geq 1$. Under this setting, Bernardo and Ledoit [2] proved a duality result that links the existence of investments with a high gain-loss ratio to pricing kernels in a single-period framework. In two previous papers, we studied this criterion in finite-state, multiperiod financial markets using the linear programming (and linear-fractional programming) duality theory [13], and in infinite state, single-period setting [14] (where ambiguity in measure is also addressed). In the present note we work in an infinite state space, multiperiod setting in the spirit of Föllmer-Schied [9]. We define a tightening of the super-hedging value through the gain-loss criterion (i.e. we obtain a price bound smaller than the superhedging value, and hence a lower and more attractive price for potential buyers of the contingent claim), which we refer to as gain-loss hedging value. We prove two duality results. The first one relates the absence of high gain-loss ratio investment strategies in infinite-state, multiperiod financial markets to the existence of a special equivalent martingale measure with bounded density and bounded away from zero. The second one is a dual representation result for the gain-loss hedging value. Such dual representations provide a starting point for the resolution of utility maximization problems in mathematical finance as illustrated in a continuous-time setting in Chapter 4 of Pham [12]. Our proof technique relies on a dual representation of the gain-loss criterion and duality theory of convex programming in infinite-dimensional spaces (see [10] for a reference on optimization in function spaces).

\section{Background}

We work in a financial market $\mathcal{M}=\left(\Omega, \mathcal{F}, \mathbb{P}, \mathbb{T}, S,\left\{\mathcal{F}_{t}\right\}_{t \in \mathbb{T}}\right)$ with discrete time trading over the time set $\mathbb{T}=\{0,1, \ldots, T\}$ and where $\left(\Omega, \mathcal{F}, \mathbb{P},\left\{\mathcal{F}_{t}\right\}_{t \in \mathbb{T}}\right)$ is a complete filtered probability space, $\mathbb{P}$ is trivial on $\mathcal{F}_{0}$, and $S=\left\{S_{t}\right\}_{t \in \mathbb{T}}$ represents the nonnegative adapted price process of stocks traded in $\mathcal{M}$ with known current prices $S_{0} \in \mathbb{R}^{d+1}$, and $S_{t} \in \mathcal{L}_{+}^{1}\left(\Omega, \mathcal{F}_{t}, \mathbb{P} ; \mathbb{R}^{d+1}\right)$ for $t=1, \ldots, T$. The first asset is the risk-free asset (the numéraire), and its price is assumed equal to one at all times $t \in \mathbb{T}$ for simplicity. A price equal to one can always be achieved for the risk-free security with a constant growth, chosen as numéraire, by dividing all other security prices and contingent claim pay-offs by the price of that security. Let us recall that in a single period setting, Bernardo and Ledoit [2] defined the gain-loss ratio $\mathbb{E}^{\mathbb{P}}\left[X_{+}\right] / \mathbb{E}^{\mathbb{P}}\left[X_{-}\right]$for a claim $X$ (where $X_{+}=\max \{0, X\}$ and $\left.X_{-}=\max \{0,-X\}\right)$ from the set $\mathcal{C}(0, S)$ of all claims that can be super-replicated at zero initial cost using the market-traded securities with prices $S$. Their key result is the duality

$$
\sup _{X \in \mathcal{C}(0, S)} \frac{\mathbb{E}^{\mathbb{P}}\left[X_{+}\right]}{\mathbb{E}^{\mathbb{P}}\left[X_{-}\right]}=\inf _{Q} \frac{\operatorname{ess} \sup \frac{\mathrm{d} Q}{\mathrm{~d} P}}{\operatorname{ess} \inf \frac{\mathrm{d} Q}{\mathrm{~d} P}}
$$

where the inf is over all equivalent martingale measures. Since the gain-loss hedging criterion is invariant under multiplication by a positive scalar, the afore-mentioned scaling does not affect the results. Additionally, we note that all equations and inequalities in the sequel involving stochastic quantities are to be understood to hold $\mathbb{P}$-a.s. 
Let $\mathcal{P}$ be the set of equivalent martingale measures in the arbitrage-free (not necessarily complete) market $\mathcal{M}$. Let $\Xi$ represent the set of $(d+1)$-dimensional predictable, self-financing portfolio processes (a.k.a. trading strategies) $\left\{\xi_{t}\right\}_{t=1, \ldots, T}$, with $\xi_{t}=\left(\xi_{t}^{0}, \xi_{t}^{1}, \ldots, \xi_{t}^{d}\right)$, and we denote $S_{t} \cdot \xi_{t}$ the inner product of vectors $S_{t}$ and $\xi_{t}$. It is well known that for a predictable trading strategy $\xi$ the following are equivalent [9]:

- $\xi$ is self-financing

- $\xi_{t} \cdot S_{t}=\xi_{t+1} \cdot S_{t}$, for $t=1, \ldots, T$

- $V_{t}=V_{0}+G_{t}=\xi_{1} \cdot S_{0}+\sum_{k=1}^{t} \xi_{k} \cdot\left(S_{k}-S_{k-1}\right)$,

where $G_{t}=\sum_{k=1}^{t} \xi_{k} \cdot\left(S_{k}-S_{k-1}\right)$ is the time $t$ component of the gains process and $V_{t}=\xi_{t} \cdot S_{t}$ is the time $t$ component of the portfolio value process $V$. Due to the presence of the numéraire (with value equal to one at all times), the knowledge of $V_{0}$ and an arbitrary $d$-dimensional predictable process $\xi$ suffice to construct the associated $(d+1)$-dimensional self-financing strategy using the above characterization. For further details we refer the reader to the opening section of Chapter 5 of [9]. For a given European contingent claim $H^{E}$ which is assumed to be an $\mathcal{F}_{T}$-measurable, $\mathbb{P}$-integrable, non-negative random variable throughout this article, the superhedging value $v^{s}$ is defined as (Definition (1) is valid even if $H^{E}$ is not a nonnegative random variable.)

$$
v^{s}=\inf \left\{x \in \mathbb{R} \mid \exists \xi \in \Xi \text { s.t. } x+\sum_{k=1}^{T} \xi_{k} \cdot\left(S_{k}-S_{k-1}\right) \geq H^{E}\right\} \text {. }
$$

Considering the position of a seller of the contingent claim, the random payment $H^{E}$ at time $T$ is a liability. Then, $v^{s}$ represents the smallest price admissible to the seller of the contingent claim since it is equal to the present value of a portfolio process $\left\{\xi_{t}\right\}_{t=1}^{T}$ whose cumulative gains $x+\sum_{k=1}^{T} \xi_{k} \cdot\left(S_{k}-S_{k-1}\right)$ dominate the payment $H^{E}$ due to the investor (the holder of the contingent claim) at time $T$, and has the smallest present value among all such portfolio processes. Therefore, $v^{s}$ is the smallest capital requirement for the seller to undertake a portfolio trading strategy which will cover the stochastic liability $H^{E}$ at maturity date $T$.

Under suitable assumptions on $H^{E}$ (e.g. $H^{E}$ bounded from below; see [8] for references and details), the following dual representation is well known (see, e.g. $[9,18])$.

THEOREM 1 The super-hedging value $v^{s}$ for a European claim $H^{E}$ has the following dual representation:

$$
v^{s}=\sup _{\mathbb{P} \in \mathcal{P}} \mathbb{E}^{\mathbb{P}}\left[H^{E}\right]
$$

In the next section we shall define a gain-loss opportunity and prove a result giving necessary and sufficient conditions for the absence of gain-loss opportunities in financial markets using duality theory of convex optimization [10]. Then, we shall pass to gain-loss hedging of European claims and establish a dual representation for the gain-loss hedging value of a European claim. 


\section{Gain-loss opportunity}

In Föllmer and Schied [9] an arbitrage opportunity is defined as a self-financing portfolio process with value process $V$ that satisfies

$$
V_{0} \leq 0, \quad V_{T} \geq 0, \quad \mathbb{P}\left[V_{T}>0\right]>0 .
$$

For $\lambda \geq 1$ we define a $\lambda$-gain-loss opportunity, $\lambda$-GLO for short, as a selffinancing portfolio process with value process $V$ satisfying

$$
V_{0}=0, \quad \mathbb{E}^{\mathbb{P}}\left[\left(V_{T}\right)_{+}-\lambda\left(V_{T}\right)_{-}\right]>0 .
$$

Since we have $S_{t} \in \mathcal{L}_{+}^{1}\left(\Omega, \mathcal{F}_{t}, \mathbb{P} ; \mathbb{R}^{d+1}\right)$ for all $t=1, \ldots, T, V_{T}$ (or $V_{T}^{-}$) is integrable $[3,5,6]$. The market has no $\lambda$-GLO (i.e. $\lambda$-GLO-free) if no such portfolio process can be found. Notice that any arbitrage opportunity is at the same time a $\lambda$-GLO for any $\lambda$. However, obviously the converse is not true. Therefore, a financial market may offer no arbitrage opportunity while for some $\lambda \geq 1$ it has $\lambda$-GLO opportunities. It is intuitive that the market admits arbitrage opportunities if and only if investments with infinite gain-loss ratio can be found (i.e. a $\lambda$-GLO with $\lambda=+\infty$ ) [2].

Example 1 Consider the example of the financial market depicted in Figure 1. The number next to each node is the value of the stock price, and the risk-free asset is assumed to be always valued at one. All scenarios are equally likely with a probability equal to $\frac{1}{6}$, i.e. the probability measure $\mathbb{P}$ is given by the vector with six components $(1 / 6, \ldots, 1 / 6)$. The financial market does not admit arbitrage opportunities $\left(\left(q_{3}, q_{4}, q_{5}, q_{6}, q_{7}, q_{8}\right)=(0.175,0.125,0.2,0.250,0.125,0.125)\right.$ is a martingale measure), but at $\lambda=1.4$ it is possible to form the following portfolio process: at $t=0$, no position is taken in the stock and the risk-free free asset, the same happens

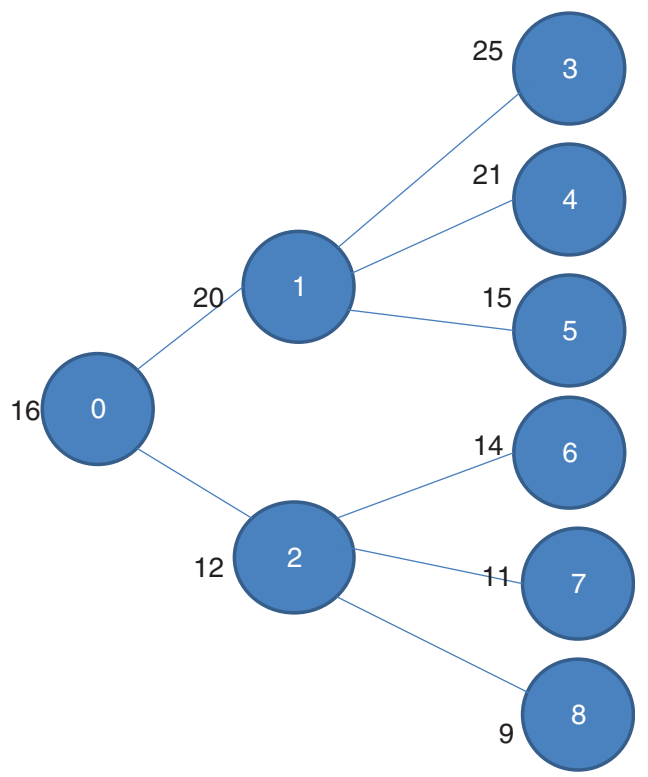

Figure 1. Example of a financial market with a stock and a risk-free asset valued at one at all time points. The number next to each node represents the value of the stock. 
at node 1 . But, at node 2, we take a short position of 5 in the stock which brings 60 units to acquire 60 units of risk-free stock. This portfolio process leaves us with a zero terminal value of $V_{T}$ ( $V_{2}$ to be precise) at nodes 3,4 and 5 . In node 7 we have a terminal value of $V_{T}$ equal to 5 , and in node 8 equal to 15 , but in node $6, V_{T}$ has value -10 . These terminal values yield exactly a gain-loss ratio equal to 1.4. The financial market does not allow the formation of portfolio processes with a gain-loss ratio equal or superior to 2 . This is due to the fact that for $\lambda \geq 2$ the maximum value of $\mathbb{E}^{\mathbb{P}}\left[\left(V_{T}\right)_{+}-\lambda\left(V_{T}\right)_{-}\right]$that can be reached using a self-financing portfolio process with value process $V$ and initial cost $V_{0}=0$ is equal to zero, attained at a trivially zero portfolio process.

One can derive a risk measure $\rho_{\lambda}: \mathcal{L}^{1} \mapsto \mathbb{R} \cup\{+\infty\}$ (to be minimized) from the gain-loss criterion as $\rho_{\lambda}\left(V_{T}\right)=\mathbb{E}^{\mathbb{P}}\left[\lambda\left(V_{T}\right)_{-}-\left(V_{T}\right)_{+}\right]$. The risk function $\rho_{\lambda}$ is always finite-valued and therefore norm-continuous [7,17]. It also satisfies monotonicity, convexity, positive homogeneity and subadditivity properties of risk measures. But, it is easy to see that it does not satisfy the translation invariance property, and the analysis of risk measures does not carry over directly as in $[9,17]$. One could certainly undertake an analysis of this function in the spirit of, e.g. [9,15-17]. However, this is beyond the scope of the present note and will be dealt with elsewhere.

The following useful dual expression can be found in [14].

Lemma 1 Let $Z \in \mathcal{L}^{1}\left(\Omega, \mathcal{F}_{T}, \mathbb{P}, \mathbb{R}\right)$ and $\lambda \geq 1$. Then, we have

$$
\mathbb{E}\left[Z_{+}\right]-\lambda \mathbb{E}\left[Z_{-}\right]=\inf \left\{\mathbb{E}[\eta Z] \mid \eta \in \mathcal{L}^{\infty}\left(\Omega, \mathcal{F}_{T}, \mathbb{P}, \mathbb{R}\right), \eta \in[1, \lambda]\right\} .
$$

Proof Define $\eta^{*}=\mathbb{1}_{\{Z>0\}}+\lambda \mathbb{1}_{\{Z \leq 0\}}$, which is $[1, \lambda]$-valued random variable, then we have

$$
\begin{aligned}
Z^{+}-\lambda Z^{-} & =Z \mathbb{1}_{\{Z>0\}}-\lambda(-Z) \mathbb{1}_{\{Z \leq 0\}} \\
& =Z \mathbb{1}_{\{Z>0\}}+\lambda Z \mathbb{1}_{\{Z \leq 0\}} \\
& =\eta^{*} Z .
\end{aligned}
$$

On the other hand, for every $[1, \lambda]$-valued random variable $\eta$ we have

$$
\begin{aligned}
\eta Z & =\eta\left(Z^{+}-Z^{-}\right)=\eta Z \mathbb{1}_{\{Z>0\}}+\eta Z \mathbb{1}_{\{Z \leq 0\}} \geq Z \mathbb{1}_{\{Z>0\}}+\lambda Z \mathbb{1}_{\{Z \leq 0\}} \\
& =\eta^{*} Z=Z_{+}-\lambda Z_{-} .
\end{aligned}
$$

We define $\mathcal{P}(\lambda)$ as the set of all martingale measures $\mathbb{Q}$ equivalent to $\mathbb{P}$ (i.e. makes the stock price process $S$ a martingale with respect to the filtration $\mathcal{F}_{t}$ ) such that the 'scaled' Radon-Nikodym density $\frac{1}{\mathcal{V}} \mathrm{d} \mathbb{Q} \mathbb{P}$ is a $\mathcal{F}_{T}$-measurable $[1, \lambda]$-valued random variable for some real number $\mathcal{V}>0$.

THEOREM 2 For fixed $\lambda \geq 1$, the market $\mathcal{M}$ is $\lambda$-GLO-free if and only if the $\operatorname{set} \mathcal{P}(\lambda)$ is non-empty.

Proof The plan of the proof is as follows. First, we shall derive a dual problem for the optimization problem to verify whether $\mathcal{M}$ is $\lambda$-GLO-free. Then using the duality theory of convex optimization we shall establish strong duality between the primal 
and dual problems. To check whether $\mathcal{M}$ is $\lambda$-GLO-free, we can solve the following problem P0:

$$
\begin{aligned}
\gamma=\sup _{z} & \left\{z \in \mathbb{R} \mid \exists \xi \in \Xi \text { s.t. } S_{0} \cdot \xi_{1}=0, \mathbb{E}\left[\sum_{k=1}^{T} \xi_{k} \cdot\left(S_{k}-S_{k-1}\right)_{+}\right]\right. \\
& \left.-\lambda \mathbb{E}\left[\sum_{k=1}^{T} \xi_{k} \cdot\left(S_{k}-S_{k-1}\right)_{-}\right] \geq z\right\},
\end{aligned}
$$

and we conclude that $\mathcal{M}$ is $\lambda$-GLO-free if $\gamma$ is zero. We can equivalently pose $\mathrm{P} 0$ as $\mathrm{P} 1$

$$
\gamma=\sup _{z, \xi \in \Xi}\left\{z \in \mathbb{R} \mid S_{0} \cdot \xi_{1}=0, \mathbb{E}\left[\sum_{k=1}^{T} \xi_{k} \cdot\left(S_{k}-S_{k-1}\right)_{+}\right]-\lambda \mathbb{E}\left[\sum_{k=1}^{T} \xi_{k} \cdot\left(S_{k}-S_{k-1}\right)_{-}\right] \geq z\right\} .
$$

The above optimization problem P1 is equivalent to the following optimization problem $\mathrm{P}$ over all self-financing (predictable) portfolio processes $\xi$

$$
\begin{array}{ll}
\sup & \mathbb{E}^{\mathbb{P}}\left[\left(S_{T} \cdot \xi_{T}\right)_{+}-\lambda\left(S_{T} \cdot \xi_{T}\right)_{-}\right] \\
\text {s.t. } & S_{0} \cdot \xi_{1}=0, \\
& S_{t} \cdot \xi_{t}=S_{t} \cdot \xi_{t+1} \quad \forall t=1, \ldots, T-1 .
\end{array}
$$

The convex programming dual problem $\mathrm{D}$ to the above optimization problem is the following problem over variables $y_{0}$ and $y_{t} \in \mathcal{L}^{\infty}\left(\Omega, \mathcal{F}_{t}, \mathbb{P} ; \mathbb{R}\right)$ for all $t=1, \ldots, T$, where $y_{T}$ is a $\left(\mathcal{F}_{T}\right.$-measurable $)[1, \lambda]$-valued random variable:

$$
\begin{aligned}
& \text { inf } 0 \\
& \text { s.t. } y_{t} S_{t}=\mathbb{E}^{\mathbb{P}}\left[y_{t+1} S_{t+1} \mid \mathcal{F}_{t}\right], \quad \forall t=0, \ldots, T-1 .
\end{aligned}
$$

We shall first derive the dual problem. To obtain the above dual problem, we form the Lagrange function using multipliers $y_{0}$ and $\left\{y_{t}\right\}_{t=1, \ldots, T-1}$ :

$$
\begin{aligned}
& L\left(\xi_{1}, \ldots, \xi_{T}, y_{0}, y_{1}, \ldots, y_{T-1}\right) \\
& \quad=\mathbb{E}^{\mathbb{P}}\left[\left(S_{T} \cdot \xi_{T}\right)_{+}-\lambda\left(S_{T} \cdot \xi_{T}\right)_{-}\right]-y_{0} S_{0} \cdot \xi_{1}+\sum_{t=1}^{T-1} \mathbb{E}^{\mathbb{P}}\left[y_{t}\left(S_{t} \cdot\left(\xi_{t}-\xi_{t+1}\right)\right] .\right.
\end{aligned}
$$

The dual function is then defined as

$$
g\left(y_{0}, y_{1}, \ldots, y_{T-1}\right)=\sup _{\xi_{1}, \ldots, \xi_{T}} L\left(\xi_{1}, \ldots, \xi_{T}, y_{0}, y_{1}, \ldots, y_{T-1}\right)
$$

with the dual problem D

$$
\inf _{y_{0}, y_{1}, \ldots, y_{T-1}} g\left(y_{0}, y_{1}, \ldots, y_{T-1}\right) .
$$

By using the dual representation of the gain-loss constraint in Lemma 1, we can equivalently re-write the dual problem as

$$
\inf _{y_{0}, y_{1}, \ldots, y_{T-1}, y_{T}} \sup _{\xi_{1}, \ldots, \xi_{T}} \mathbb{E}^{\mathbb{P}}\left[y_{T}\left(S_{T} \cdot \xi_{T}\right)\right]-y_{0} S_{0} \cdot \xi_{1}+\sum_{t=1}^{T-1} \mathbb{E}^{\mathbb{P}}\left[y_{t}\left(S_{t} \cdot\left(\xi_{t}-\xi_{t+1}\right)\right],\right.
$$


where $y_{T}$ is an $\mathcal{F}_{T}$-measurable, $[1, \lambda]$-valued random variable. Now, to evaluate the inner supremum, we re-write the function using conditional expectations and after re-grouping the terms concerning each $\xi_{t}$ :

$$
\begin{aligned}
\mathbb{E}^{\mathbb{P}}\left[\mathbb{E}^{\mathbb{P}}\left[\left(y_{T} S_{T}-y_{T-1} S_{T-1}\right) \cdot \xi_{T} \mid \mathcal{F}_{T-1}\right]\right. \\
\left.\quad+\cdots+\mathbb{E}^{\mathbb{P}}\left[\left(y_{2} S_{2}-y_{1} S_{1}\right) \cdot \xi_{2} \mid \mathcal{F}_{1}\right]+\left(y_{1} S_{1}-y_{0} S_{0}\right) \cdot \xi_{1}\right] .
\end{aligned}
$$

Now, the supremum does not blow up, provided that

$$
y_{t} S_{t}=\mathbb{E}^{\mathbb{P}}\left[y_{t+1} S_{t+1} \mid \mathcal{F}_{t}\right], \quad \forall t=0, \ldots, T-1,
$$

which are exactly the constraints of the dual problem with an identically zero objective function.

Now, assume problem $\mathrm{P}$ has an optimal value of zero, i.e. $\mathcal{M}$ is $\lambda$-GLO-free, and an optimal solution $\xi^{*}$ (all portfolio positions identically zero achieves this zero objective function value so we can always assume that the set of optimal solutions $\operatorname{sol}(P)$ is non-empty in this case). By the Lagrange Duality Theorem (Theorem 1 in Section 8.6 of [10]) we have that $\sup (P)=\inf (D)$ since it is easy to see that $P 1$ is equivalent to $\mathrm{P}$, and $\mathrm{P} 0$ satisfies the Slater strict feasibility condition. As a result, the dual equations must have a solution $y_{0},\left\{y_{t}\right\}_{t=1, \ldots, T}$ (since otherwise the dual objective function value would be $+\infty$ ). The dual equations are equivalently written as (after scaling)

$$
S_{t}=\mathbb{E}^{\mathbb{Q}}\left[S_{t+1} \mid \mathcal{F}_{t}\right], \quad \forall t=0, \ldots, T-1,
$$

where $\mathbb{Q} \in \mathcal{P}(\lambda)$. This completes the proof of necessity.

For the converse, let us assume that the set $\mathcal{P}(\lambda)$ is non-empty. As a consequence, the system of equations in $\left\{y_{t}\right\}$

$$
y_{t} S_{t}=\mathbb{E}^{\mathbb{P}}\left[y_{t+1} S_{t+1} \mid \mathcal{F}_{t}\right], \quad \forall t=0, \ldots, T-1
$$

has a solution, which implies by the weak duality theorem of convex optimization $(\sup (P) \leq \inf (D))$ that $\sup (P)=0$ and the sup is in fact attained (by the always zero portfolio process). Therefore, the market is $\lambda$-GLO-free.

\section{The dual representation for gain-loss hedging}

For a fixed $\lambda \geq 1$ we define the gain-loss hedging value $v^{g l}$ for a contingent claim $H^{E} \in \mathcal{L}_{+}^{1}\left(\Omega, \mathcal{F}_{t}, \mathbb{P} ; \mathbb{R}\right)$ as

$$
\begin{aligned}
v^{g l}=\inf \left\{x \in \mathbb { R } | \exists \xi \in \Xi \text { s.t. } \mathbb { E } ^ { \mathbb { P } } \left[\left(x+\sum_{k=1}^{T} \xi_{k} \cdot\left(S_{k}-S_{k-1}\right)-H^{E}\right)_{+}\right.\right. \\
\left.\left.-\lambda\left(x+\sum_{k=1}^{T} \xi_{k} \cdot\left(S_{k}-S_{k-1}\right)-H^{E}\right)_{-}\right] \geq 0\right\} .
\end{aligned}
$$

The gain-loss hedging value represents the present value of an optimal portfolio process whose value at time $T$ dominates the value of the contingent claim in the following sense: the expected value of surplus generated by the portfolio process over the contingent claim payment is at least as large as the $\lambda$ multiple of expected 
shortage with respect to the contingent claim payment. Among such portfolios, the one resulting in the smallest present value is sought. The optimization problem in (6) is a relaxation of the superhedging problem (1) since every predictable, self-financing portfolio process that satisfies the constraints of the problem defining $v^{s}$ in the previous section also satisfies the above gain-loss constraint. Therefore, we have $v^{g l} \leq v^{s}$.

As a consequence of Lemma 1, the gain-loss constraint

$$
\mathbb{E}^{\mathbb{P}}\left[\left(x+\sum_{k=1}^{T} \xi_{k} \cdot\left(S_{k}-S_{k-1}\right)-H^{E}\right)_{+}-\lambda\left(x+\sum_{k=1}^{T} \xi_{k} \cdot\left(S_{k}-S_{k-1}\right)-H^{E}\right)_{-}\right] \geq 0
$$

is equivalent to

$$
\begin{aligned}
& \mathbb{E}^{\mathbb{P}}\left[\eta\left(x+\sum_{k=1}^{T} \xi_{k} \cdot\left(S_{k}-S_{k-1}\right)-H^{E}\right)\right] \geq 0 \\
& \quad \forall \mathcal{F}_{T} \text {-meas. }[1, \lambda] \text {-valued random variables } \eta
\end{aligned}
$$

Definition 1 The European claim $H^{E}$ is called $\lambda$-attainable if the gain-loss hedging value $v^{g l} \equiv V_{0}$ is a finite non-negative real number.

The notion of $\lambda$-attainability may be viewed as an extension of the concept of exact replication for a contingent claim, relaxed using the gain-loss criterion. More precisely, the requirement that all possible pay-offs at time $T$ be replicated by a portfolio almost surely (the present value of such a portfolio process is then the fair price of the contingent claim since it does not allow arbitrage) is replaced by the requirement that one finds a portfolio process which replicates a given contingent claim in the sense of Definition 1, i.e. the value of the portfolio process in excess of the contingent claim $H^{E}$ is equal to or larger than in expectation to a $(\lambda)$ multiple of the value falling short of $H^{E}$ in expectation times. The value at time $t=0$ of such a portfolio process is then taken as a 'fair' price for the contingent claim, in the sense of not allowing gain-loss ratio opportunities superior to $\lambda$ in the financial market.

If the contingent claim in question is not $\lambda$-attainable, then by definition $v^{g l}$ is either $+\infty$ or $-\infty$. We shall now show that the case $+\infty$ is impossible by demonstrating that an admissible solution can always be found for the optimization problem (6). Let $\xi \in \Xi$ be any self-financing, predictable portfolio strategy and denote by $Z^{\xi}$ the quantity $\sum_{k=1}^{T} \xi_{k} \cdot\left(S_{k}-S_{k-1}\right)-H^{E}$. Now achieving feasibility means finding an $x \in \mathbb{R}$ such that

$$
\mathbb{E}^{\mathbb{P}}\left[\left(x+Z^{\xi}\right)_{+}\right]-\lambda \mathbb{E}^{\mathbb{P}}\left[\left(x+Z^{\xi}\right)_{-}\right] \geq 0 .
$$

But by Lemma 1, the above inequality is equivalent to

$$
\mathbb{E}^{\mathbb{P}}\left[\eta\left(x+Z^{\xi}\right)\right] \geq 0, \quad \text { for all }[1, \lambda] \text {-valued, } \mathcal{F}_{T} \text {-measurable } \eta \text {. }
$$

We can rewrite the last inequality as

$$
\mathbb{E}^{\mathbb{P}}\left[\eta\left(-Z^{\xi}\right)\right] \leq x \mathbb{E}^{\mathbb{P}}[\eta], \quad \text { for all }[1, \lambda] \text {-valued, } \mathcal{F}_{T} \text {-measurable } \eta,
$$


or, equivalently as

$$
\mathbb{E}^{\mathbb{P}}\left[\frac{\eta}{\mathbb{E}^{\mathbb{P}}[\eta]}\left(-Z^{\xi}\right)\right] \leq x, \quad \text { for all }[1, \lambda] \text {-valued, } \mathcal{F}_{T^{-} \text {-measurable } \eta}
$$

Now, we have

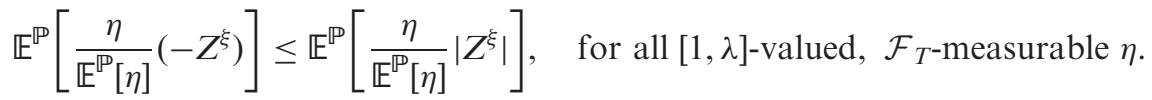

Since $\eta \in[1, \lambda]$, one has $1 \leq \mathbb{E}^{\mathbb{P}}[\eta] \leq \lambda$. Hence, we get $\frac{1}{\lambda} \leq \frac{\eta}{\mathbb{E}^{P}[\eta]} \leq \lambda$. Therefore, we obtain

$$
\mathbb{E}^{\mathbb{P}}\left[\frac{\eta}{\mathbb{E}^{\mathbb{P}}[\eta]}\left|Z^{\xi}\right|\right] \leq \lambda \mathbb{E}^{\mathbb{P}}\left[\left|Z^{\xi}\right|\right] \text { for all }[1, \lambda] \text {-valued, } \mathcal{F}_{T} \text {-measurable } \eta .
$$

As a result, for feasibility it suffices to choose $x \in \mathbb{R}$ such that

$$
x \geq \lambda \mathbb{E}^{\mathbb{P}}\left[\left|Z^{\xi}\right|\right] .
$$

On the other hand, if $v^{g l}=-\infty$ then we can find a predictable, self-financing portfolio process $\xi \in \Xi$ with value process $V$, such that

$$
\mathbb{E}^{\mathbb{P}}\left[\left(V_{0}+\sum_{k=1}^{T} \xi_{k} \cdot\left(S_{k}-S_{k-1}\right)-H^{E}\right)_{+}-\lambda\left(V_{0}+\sum_{k=1}^{T} \xi_{k} \cdot\left(S_{k}-S_{k-1}\right)-H^{E}\right)_{-}\right]>0
$$

with $V_{0}=0$. We can rewrite the last inequality as

$$
\begin{aligned}
& \mathbb{E}^{\mathbb{P}}\left[\eta\left(V_{0}+\sum_{k=1}^{T} \xi_{k} \cdot\left(S_{k}-S_{k-1}\right)-H^{E}\right)\right]>0, \\
& \forall \mathcal{F}_{T} \text {-meas. }[1, \lambda] \text {-valued random variables } \eta,
\end{aligned}
$$

or, equivalently

$$
\begin{aligned}
& \mathbb{E}^{\mathbb{P}}\left[\eta\left(V_{0}+\sum_{k=1}^{T} \xi_{k} \cdot\left(S_{k}-S_{k-1}\right)\right)\right]>\mathbb{E}^{\mathbb{P}}\left[\eta H^{E}\right], \\
& \forall \mathcal{F}_{T} \text {-meas. }[1, \lambda] \text {-valued random variables } \eta .
\end{aligned}
$$

But, we have $\mathbb{E}^{\mathbb{P}}\left[\eta H^{E}\right] \geq 0$ for all $\mathcal{F}_{T}$-measurable $[1, \lambda]$-valued random variables $\eta$ since $H^{E}$ is a non-negative random variable. Therefore, we have

$$
\begin{aligned}
& \mathbb{E}^{\mathbb{P}}\left[\eta\left(V_{0}+\sum_{k=1}^{T} \xi_{k} \cdot\left(S_{k}-S_{k-1}\right)\right)\right]>0, \\
& \quad \forall \mathcal{F}_{T} \text {-meas. }[1, \lambda] \text {-valued random variables } \eta,
\end{aligned}
$$

or, equivalently

$$
\mathbb{E}^{\mathbb{P}}\left[\left(V_{0}+\sum_{k=1}^{T} \xi_{k} \cdot\left(S_{k}-S_{k-1}\right)\right)_{+}-\lambda\left(V_{0}+\sum_{k=1}^{T} \xi_{k} \cdot\left(S_{k}-S_{k-1}\right)\right)_{-}\right]>0,
$$

with $V_{0}=0$. Hence, the market is not $\lambda$-GLO free. Therefore, the set $\mathcal{P}(\lambda)$ is empty by Theorem 2. 
Now, assume the market is $\lambda$-GLO free (and hence arbitrage-free) and any European contingent claim satisfying our assumptions is $\lambda$-attainable. We can rewrite the problem (6) as the following optimization problem (P2):

$$
\begin{array}{ll}
\inf & S_{0} \cdot \xi_{1} \\
\text { s.t. } & S_{t} \cdot\left(\xi_{t+1}-\xi_{t}\right)=0 \quad \forall t=1, \ldots, T-1, \\
& \mathbb{E}^{\mathbb{P}}\left[\left(S_{T} \cdot \xi_{T}-H^{E}\right)_{+}-\lambda\left(S_{T} \cdot \xi_{T}-H^{E}\right)_{-}\right] \geq 0,
\end{array}
$$

where $\mathrm{P} 2$ is a convex, semi-infinite optimization problem. Without repeating the details of the computation (they are very similar to those in the proof of Theorem 2) we obtain the convex programming dual of $\mathrm{P} 2$ as the following problem D2, over positive dual variables $\left\{y_{t}\right\}_{t=1, \ldots, T}$ such that $y_{t} \in \mathcal{L}^{\infty}\left(\Omega, \mathcal{F}_{t}, \mathbb{P} ; \mathbb{R}\right)$ for all $t=1, \ldots, T-1$, with $\eta$ an $\mathcal{F}_{T}$-measurable $[1, \lambda]$-valued (random) dual variable:

$$
\begin{array}{ll}
\text { sup } & y_{T} \mathbb{E}^{\mathbb{P}}\left[\eta H^{E}\right] \\
\text { s.t. } & S_{0}=\mathbb{E}^{\mathbb{P}}\left[y_{1} S_{1} \mid \mathcal{F}_{1}\right], \\
& y_{t} S_{t}=\mathbb{E}^{\mathbb{P}}\left[y_{t+1} S_{t+1} \mid \mathcal{F}_{t}\right], \quad \forall t=1, \ldots, T-2 \\
& y_{T-1} S_{T-1}=y_{T} \mathbb{E}^{\mathbb{P}}\left[\eta S_{T} \mid \mathcal{F}_{T-1}\right] .
\end{array}
$$

According to the duality theory of convex programming (Theorem 1, Section 8.6 of [10]), we have $\inf (P 2)=\sup (D 2)$ and the sup in (D2) is attained since (P2) satisfies the Slater strict feasibility condition. Finally, it remains to observe that the dual problem (D2) is equivalent to the following problem by a straightforward scaling transformation using $y_{T}$ :

$$
\sup _{\mathbb{P}^{*} \in \mathcal{P}(\lambda)} \mathbb{E}^{*}\left[H^{E}\right]
$$

Therefore, we get

$$
v^{g l}=\max _{\mathbb{P}^{*} \in \mathcal{P}(\lambda)} \mathbb{E}^{*}\left[H^{E}\right]
$$

Hence, we have proved the following main result of the present note.

THEOREM 3 In a $\lambda$-GLO-free market $\mathcal{M}$ all European contingent claims $H^{E}$ are $\lambda$-attainable, and the gain-loss hedging value $v^{g l}$ admits the dual representation (7), i.e. we have the duality relation

$$
\begin{aligned}
\max _{\mathbb{P}^{*} \in \mathcal{P}(\lambda)} \mathbb{E}^{*}\left[H^{E}\right]=\inf & \left\{x \in \mathbb { R } | \exists \xi \in \Xi \text { s.t. } \mathbb { E } ^ { \mathbb { P } } \left[\left(x+\sum_{k=1}^{T} \xi_{k} \cdot\left(S_{k}-S_{k-1}\right)-H^{E}\right)_{+}\right.\right. \\
& \left.\left.-\lambda\left(x+\sum_{k=1}^{T} \xi_{k} \cdot\left(S_{k}-S_{k-1}\right)-H^{E}\right)_{-}\right] \geq 0\right\} .
\end{aligned}
$$

The following example illustrates the fact that the gain-loss hedging value can be smaller than the superhedging value.

Example 2 Consider the financial market of Example 1, and a European call option with strike equal to 11 . The superhedging value $v^{s}$ of the option is 5.4 , and it is 
obtained by evaluating the expectation of the option pay-off at the martingale measure given by $\left(q_{3}, q_{4}, q_{5}, q_{6}, q_{7}, q_{8}\right)=(0,0.417,0.083,0.3,0,0.2)$. Evaluating $v^{g l}$ at $\lambda=2$, we obtain a price equal to 5.25 attained at the measure $\left(q_{3}, q_{4}, q_{5}, q_{6}, q_{7}, q_{8}\right)=$ $(0.125,0.208,0.167,0.250,0.125,0.125)$ which belongs to $\mathcal{P}(2)$ with $\mathcal{V}=3 / 4$.

It was pointed out by an anonymous referee that the results of this article can be re-derived in the framework of continuous time trading departing from the existing results on superreplication of sufficiently integrable claims, representation of risk measures and hedging with risk measures given in $[1,4,7,11]$. We shall undertake this endeavour in a future paper.

\section{Acknowledgements}

Author was supported in part by a visiting researcher grant from the University of L'Aquila, Italy, made possible by Research Grant Reti per la conoscenza e l'orientamento tecnicoscientifico per lo sviluppo della competitività (RE.C.O.TE.S.S.C.) POR 2007-2013-Action 4, funded by Regione Abruzzo and the European Social Fund 2007-2013. The author is grateful to Savaş Dayanık for useful discussions and to an anonymous referee for a careful review.

\section{References}

[1] P. Barrieu and N. El Karoui, Pricing, hedging and optimally designing derivatives via minimization of risk measures, in Indifference Pricing: Theory and Applications, R. Carmona, ed., Princeton University Press, Princeton, USA, 2008, pp. 77-146.

[2] A.E. Bernardo and O. Ledoit, Gain, loss and asset pricing, J. Political Econ. 108 (2000), pp. 144-172.

[3] S. Biagini and A. Cerny, Admissible strategies in semimartingale portfolio selection, SIAM J. Control Optim. 49 (2011), pp. 42-72.

[4] S. Biagini and M. Frittelli, On the superreplication price of unbounded claims, Ann. Appl. Probab. 14 (2004), pp. 1970-1991.

[5] S. Biagini and M. Frittelli, Utility maximization in incomplete markets for unbounded processes, Finance Stoch. 9 (2005), pp. 493-517.

[6] S. Biagini and M. Frittelli, A unified framework for utility maximization problems: An Orlicz space approach, Ann. Appl. Probab. 18 (2008), pp. 929-966.

[7] S. Biagini and M. Frittelli, On the extension of the Namioka-Klee theorem and the Fatou property for risk measures, in Optimality and Risk: Modern Trends in Mathematical Finance: The Kabanov Festschrift, F. Delbaen, M. Rasonyi, and Ch. Stricker, eds., Springer, Berlin, 2009, pp. 1-28.

[8] F. Delbaen and W. Schachermayer, The Mathematics of Arbitrage, Springer-Verlag, Berlin, 2006.

[9] H. Föllmer and A. Schied, Stochastic Finance: An Introduction in Discrete Time, De Gruyter Studies in Mathematics, 2nd ed., Walter de Gruyter, Berlin, 2004.

[10] D.G. Luenberger, Optimization by Vector Space Methods, John Wiley \& Sons, New York, 1969.

[11] F. Oertel and M. Owen, On utility-based superreplication prices of contingent claims with unbounded payoffs, J. Appl. Probab. 44 (2007), pp. 880-888.

[12] H. Pham, Continuous-time Stochastic Control and Optimization with Financial Applications, Springer-Verlag, Berlin, 2009.

[13] M.Ç. Pınar, A. Altay-Salih, and A. Camc1, Expected gain-loss pricing and hedging of contingent claims in incomplete markets by linear programming, Eur. J. Oper. Res. 201 (2010), pp. $770-785$. 
[14] M.Ç. Pınar, Gain-loss pricing under ambiguity of measure, ESAIM: Control, Optim. Calc. Var. 16 (2010), pp. 132-147.

[15] B. Rudloff, Convex hedging in incomplete markets, Appl. Math. Finance 14 (2007), pp. $437-452$.

[16] B. Rudloff, Coherent hedging in incomplete markets, Quant. Finance 9 (2009), pp. 197-206.

[17] A. Ruszczyński and A. Shapiro, Optimization of convex risk functions, Math. Oper. Res. 31 (2006), pp. 433-452.

[18] M. Schäl, Martingale measures and hedging for discrete-time financial markets, Math. Oper. Res. 24 (1999), pp. 509-528. 